\title{
METOdOLOGÍAS DE ANÁLISIS DE CADENAS PRODUCTIVAS CON ENFOQUE PARTICIPATIVO Y DE CADENA DE VALOR: ANÁLISIS COMPARATIVO
}

\author{
Manuela Mercedes Pendón (Universidad Nacional de La Plata, Argentina) manue- \\ la.pendon@ing.unlp.edu.ar \\ Eduardo Williams (Universidad Nacional de La Plata, Argentina) williams@ing.unlp.edu.ar \\ Natalia Cibeira (Universidad Nacional de La Plata, Argentina) naty_cib@msn.com \\ Alejo Castroman (Universidad Nacional de La Plata, Argentina) alejocastroman8@hotmail.com
}

Resumen: Este trabajo presenta un análisis comparativo entre las metodologías de análisis de cadenas productivas con enfoque participativo y de cadena de valor. Se presentan conceptos teóricos, una investigación bibliográfica sobre las distintas metodologías, y finalmente una comparación teniendo en cuenta diversos factores como cantidad de etapas, profundidad, duración y aplicaciones existentes.

Palabras clave: Cadena de suministro. Cadena de valor. Producción.

\section{Introducción}

En años recientes, el fenómeno económico y social de la Globalización dio lugar a la apertura de los mercados con la consecuente creación de un mercado global de productos y manufacturas que compiten a escala mundial y el desafío a la empresas de ser cada vez más eficientes y cumplir los estándares de calidad para satisfacer las demandas de productos a nivel nacional e internacional. En este contexto, se observa que gobiernos y organismos, a distintos niveles, de todo el mundo, se encuentran trabajando en planes estratégicos industriales, para promover sus industrias y mejorar la competitividad de sus empresas y regiones e inclusive como herramientas para erradicar la pobreza. Es en estos estudios donde se observa la utilización de los términos Cadena Productiva, Cadena de Suministro y Cadena de Valor, y se presentan distintas metodologías para su análisis. Aparece allí la necesidad de comparar las metodologías existentes a través de determinados factores para facilitar la elección de alguna de ellas para su aplicación algún caso particular o tomar como base para seguir construyendo en este sentido.

2. Definiciones: Cadena Productiva, Cadena de Suministro y Cadena de Valor 


\section{ReLAlnEP}

Cadena productiva es el conjunto de operaciones necesarias para llevar a cabo la producción de un bien o servicio, que ocurren de forma planificada, y producen un cambio o transformación de materiales, objetos o sistemas. Una cadena productiva consta de etapas consecutivas a lo largo de las que diversos insumos sufren algún tipo de transformación, hasta la constitución de un producto final y su colocación en el mercado.

Se trata, por tanto de una sucesión de operaciones de diseño, producción y de distribución integradas, realizadas por diversas unidades interconectadas como una corriente, involucrando una serie de recursos físicos, tecnológicos, económicos y humanos. La cadena productiva abarca desde la extracción y proceso de manufacturado de la materia prima hasta el consumo final.

Según Secretaría Técnica ASOCAM Intercooperation (2012) (Figura 1) una cadena productiva es "un sistema constituido por actores interrelacionados y por una sucesión de operaciones, de producción, transformación, comercialización de un producto, en un entorno determinado".

El análisis de la misma permite una comprensión sistémica de las relaciones entre los actores involucrados en el proceso que sigue un producto con el objetivo de que los diferentes actores interesados en colocar con éxito el producto en el mercado, pueden detectar los problemas, cuellos de botella o factores críticos que bloquean determinado eslabón de la cadena.

\section{FIGURA 1: CADENA PRODUCTIVA}

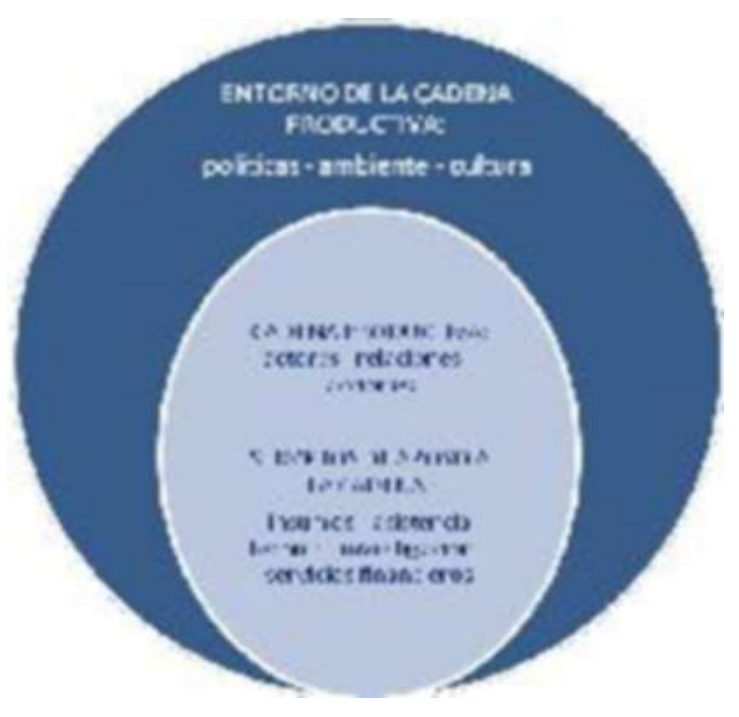

Fuente: Secretaría Técnica ASOCAM. Intercooperation

Una cadena de suministro está formada por todas aquellas partes involucradas de manera directa o indirecta en la satisfacción de la solicitud de un cliente. La cadena de suministro incluye no solamente al fabricante y al proveedor, sino también a los transportistas, almacenistas, vendedores al detalle (o menudeo) e incluso a los mismos clientes.

Dentro de cada organización, como la del fabricante, abarca todas las funciones que participan en la recepción y el cumplimiento de una petición del cliente. Estas funciones incluyen, pero no están limitadas al desarrollo de nuevos productos, la mercadotecnia, las operaciones, la distribución, las finanzas y el servicio al cliente. (CHOPRA; MEINDL, 2006).

La cadena de suministro es la secuencia de eventos que cubren el ciclo de vida entero de 


\section{ReLAlnEP}

un producto o servicio desde que es concebido hasta que es consumido (BLANCHARD, 2010).

El término cadena de suministro no está limitado a empresas manufactureras, sino que se ha ampliado para incluir tanto productos tangibles como servicios intangibles que llegan al consumidor (AYERS, 2000).

Según el Consejo de Profesionales de Administración de Cadena de Suministro (CSCMP) la Cadena de Suministro eslabona a muchas compañías, iniciando con materias primas no procesadas y terminando con el consumidor final utilizando los productos terminados. Todos los proveedores de bienes y servicios y todos los clientes están eslabonados por la demanda de los consumidores de productos terminados al igual que los intercambios materiales e informáticos en el proceso logístico, desde la adquisición de materias primas hasta la entrega de productos terminados al usuario final.

Históricamente, el término cadena de suministro surgió cuando Keith Oliver, un consultor en Booz Allen Hamilton, lo utilizó en una entrevista para el Financial Times en el año 1982. A partir de allí fue tomando fuerza en el ambiente de los negocios y comenzó a estar presente en publicaciones sobre el tema, convirtiéndose en un término regular en administración e ingeniería.

Años mas tarde surge la denominada Administración de la Cadena de Suministro (SCM: Supply Chain Management), que puede definirse como una filosofía administrativa continua y evolutiva que busca unificar los recursos productivos totales de las funciones de negocio de la empresa y sus socios aliados a lo largo de toda la Cadena de Suministro, buscando un sistema altamente competitivo enfocado a desarrollar soluciones innovadoras y a sincronizar el flujo de los productos, servicios e información hacia el mercado, creando un valor único e individualizado para el cliente (ROSS, 1996).

Se entiende que este concepto aplica a empresas medianas y grandes que han superado ya la etapa de crecimiento en su curva de madurez, ya que presupone la existencia y práctica de conceptos como Calidad Total, Servicio, Control de Procesos y Mantenimiento Productivo Total. Sin embargo en Latinoamérica y en general en los países del tercer mundo el reconocimiento de la necesidad no se ha arraigado aún en el pensamiento de los ejecutivos y administradores de sus empresas. Actualmente al escuchar hablar de "SCM" lo relacionan con el tema de la Reingeniería, cuestionando su utilidad.

El objetivo de la SCM, según varios autores de revistas y/o artículos de investigación, es incrementar el servicio a los clientes y simultáneamente reducir costos, para lograrlo es necesario reducir los recursos utilizados para dicho propósito, principalmente inventarios.

Sus principios fundamentales son:

- Administración de inventarios a través de todo la cadena

- Relaciones a largo plazo

- Compartir información

- Integración/coordinación

- Enfoque en el cliente final 
- Visión de la cadena de suministro total como una sola entidad

- Relaciones ganar/ganar.

La cadena de valor empresarial, o cadena de valor, es un modelo teórico que permite describir el desarrollo de las actividades de una organización empresarial generando valor al cliente final, descrito y popularizado por Michael Porter en su obra Competitive Advantage: creating and sustaining superior performance (PORTER, 1985).

La cadena de valor es una herramienta de análisis para planificación estratégica. Su objetivo último es maximizar la creación de valor mientras se minimizan los costes. De lo que se trata es de crear valor para el cliente, lo que se traduce en un margen entre lo que se acepta pagar y los costos incurridos por adquirir la oferta. Sin embargo, la práctica ha demostrado que la reducción de costos monetarios tiene también un límite tecnológico, pues en ocasiones ha afectado también la calidad de la oferta y el valor que ésta genera. Por ello el pensamiento sistémico en este aspecto ha evolucionado a desarrollar propuestas de valor, en las que la oferta se diseña integralmente para atender de modo óptimo a la demanda (CEDILLO, SANCHEZ; SANCHEZ, 2006).

\section{El enfoque de Cadena de Valor}

El concepto de Cadena de Valor ha sido extendido más allá de las organizaciones individuales. También puede ser aplicado al estudio de la cadena de suministro así como a redes de distribución. La puesta a disposición de un conjunto de productos y servicios al consumi- dor final moviliza diferentes actores económicos, cada uno de los cuales gestiona su cadena de valor. Las interacciones sincronizadas de esas cadenas de valor locales crean una cadena de valor ampliada que puede llegar a ser global. Capturar el valor generado a lo largo de la cadena es la nueva aproximación que han adoptado muchos estrategas de la gestión. A base de explotar la información que se dirige hacia arriba y hacia abajo dentro de la cadena, las compañías pueden intentar superar los intermediarios creando nuevos modelos de negocio.

En particular, en Argentina y otros países latinoamericanos el término es actualmente usado para incluir a todos los sectores productivos que intervienen en la generación de un bien o servicio. El concepto es afín, pero no sinónimo, al de encadenamiento productivo y es por ese motivo que surge en algunos casos el uso indistinto de uno u otro termino.

El concepto de cadena de valor es actualmente utilizado para discutir el problema del desarrollo de producciones locales y regionales que puedan mejorar el ingreso de poblaciones marginadas. (KAPLINSKY; MORRIS, 2000). Este análisis permitiría (MITCHEL; COLES; KEANE, 2009):

- Identificar factores críticos que condicionan el acceso a determinados bienes y servicios, identificando los sectores en los que se requieren intervenciones estatales.

- Determinar la viabilidad económica y comercial de emprendimientos. 


\section{ReLAInEPI}

- Identificar los beneficiados y perjudicados en las distintas etapas y sectores de los encadenamientos productivos.

- Obtener un análisis independiente de la escala productiva, por ser un método inherentemente aplicable tanto a productores individuales como a grupos de ellos, a una localidad o región como a todo un país.

- Minimizar la influencia de modelos económicos al basarse casi exclusivamente en datos económicos de medición directa.

- Formular acciones concretas para contrarrestar tanto efectos indeseables del mercado como de las regulaciones estatales.

- Tener explícitamente en cuenta la indefensión de los actores más débiles frente a reglas de juego fijadas por los actores poderosos de la cadena, y la restricción que esto impone a las elecciones de los primeros.

El análisis de cadenas es solo una herramienta de análisis que permite identificar los principales puntos críticos que frenan la competitividad de un producto, para luego definir e impulsar estrategias concertadas entre los principales actores involucrados (PLATAFORMA RURALTER, 2004).

En Fundación CODESPA (2011) se realiza la distinción entre cadenas productivas y el enfoque de cadenas de valor, en este sentido, el documento expresa que "Las cadenas productivas o cadenas de producción existen en todas partes, pero no siempre bajo un enfoque de cadena de valor.

Este último constituye un marco de análisis integral (desde la provisión de insumos hasta la comercialización) orientado a mejorar la competitividad y equidad en las cadenas productivas. Analiza el contexto, los actores (el rol que juegan y sus relaciones), los puntos críticos así como las principales barreras de participación, acceso a servicios de apoyo y recursos por parte de personas en riesgo de exclusión.

A partir de ahí, se diseña una estrategia o plan de acción con el que se busca añadir un valor económico y social sostenible para las personas más pobres que forman parte de la cadena y lograr un impacto más sostenible.” Y plantea una comparación entre ambos términos, la cual se resume en el siguiente Cuadro 1.

\section{CUADRO 1: DISTINCIÓN ENTRE CADENAS PRODUCTIVAS Y EL ENFOQUE DE CADENAS DE}

VALOR

\begin{tabular}{|c|c|c|}
\hline Estructura Organizativa & Actores independientes & Actores dependientes \\
\hline Orientación & Liderado por la oferta & Liderado por la demanda \\
\hline Identificación de mercado & Potencial de mercado & Nicho y negocios concretos \\
\hline Elemento Principal & Costo/precio & Valor/calidad \\
\hline Estrategia & Productos básicos & Productos diferenciados \\
\hline Relación entre los actores & Informal & Formal \\
\hline
\end{tabular}




\begin{tabular}{|c|c|c|}
\hline Estructura Organizativa & Actores independientes & Actores dependientes \\
\hline Visión de la relación & Corto Plazo & Largo Plazo \\
\hline Nivel de confianza & Bajo/medio & Alto \\
\hline Flujo de la información & Escasa o ninguna & Amplia \\
\hline
\end{tabular}

Fuente: Elaboración propia en base a Fundación CODESPA (2011)

Surge de la comparativa entre cadenas productivas y cadenas de valor que las primeras tienen en general una visión poco clara del mercado, con imposibilidad de afectar directamente al producto. Se maneja información genérica que no aporta datos relevantes para la toma de decisiones estratégicas teniendo en cuenta características específicas de la demanda.

Por el contrario, a través del enfoque de cadena de valor, los actores involucrados manejan información concreta sobre el mercado del producto y pueden identificar concretamente cuales son los productos esperados por los consumidores finales.

Esta característica resulta en una ventaja competitiva para la cadena ya que trabajando sobre las especificaciones de los clientes sobre el producto todos los actores de la cadena trabajan en pos de su obtención, lo cual, a su vez, se convierte en mayor incentivo para trabajar de forma coordinada y mejorar la competitividad, a través de acciones que faciliten el desarrollo de productos de calidad, nuevos nichos de mercado y sostenibilidad de la cadena.

Dentro de esta última deben incluirse no solo la sostenibilidad en términos económicos sino también la sostenibilidad desde el punto de vista ambiental y social como factores destacados en el desarrollo de cadenas de valor.

Por otro lado, una cadena de valor es más competitiva cuando sus diferentes actores han desarrollado relaciones eficientes y equitativas entre ellos. Esto significa que los diferentes actores se han organizado, coordinan y comparten información; tienen intereses diferentes pero comparten una visión común del desarrollo de la cadena; producen en función de una demanda de mercado; se diferencian por elaborar productos de calidad e innovadores; aprovechan oportunidades de mercado y; son eficientes en costos. El enfoque de cadena de valor es un campo adecuado para cultivar todos estos retos, que no siempre se cumplen en una cadena productiva.

Es importante que en el enfoque de cadena de valor se generen relaciones equitativas y un reparto de beneficios justo entre los actores. Para ello, todos deben tener acceso a información y recursos, tener poder de decisión y negociación, y recibir beneficios acorde con su inversión, el trabajo realizado y el riesgo asumido.

Para que la cadena pueda ser capaz de hacer frente a la realidad dinámica y compleja y a los cambios constantes del mercado y el entorno, se deben promover las relaciones horizontales y cercanas entre sus miembros para que, por ejemplo, haya fluidez de información entre sus eslabones.

Éste es un tema fundamental en las cadenas de valor y a la vez uno de los mayores retos. La información en la cadena productiva es parte 
del poder que tienen los intermediarios o el actor encargado de la comercialización final; información que desconoce la pequeña empresa del productor, con lo que, en muchas ocasiones, su poder de negociación o de voz es reducido.

Como se ha comentado previamente, las cadenas de valor tienen una visión a largo plazo, en el que se intentan lograr negocios futuros, porque existe un alto nivel de confianza entre todos los actores. Otro de los factores necesarios en las cadenas de valor es un nivel de confianza muy alto entre los actores.

\section{Metodologías de Análisis de Cadenas} Productivas con carácter participativo

Las metodologías que se resumen a continuación están basadas en el uso de métodos participativos. Dichos métodos, que incluyen grupos focales, mapeos, y otras formas de reflexión facilitada dan prioridad no solamente a los resultados de su aplicación sino al proceso de aplicación como tal. En este sentido, si bien se genera un resultado concreto (un mapa, una matriz, una tabla, etc.) este no es el objetivo del ejercicio sino el proceso de análisis y reflexión que se genera alrededor de la herramienta metodológica.

En la literatura pueden encontrarse distintas metodologías de análisis de cadenas productivas con carácter participativo. Dentro de ellas pueden mencionarse las más relevantes, a saber:
- Metodología de RURALTER (PLATAFORMA RURALTER, 2004).

- Metodología del CIP (Centro Internacional de la Papa) (BERNET; THIELE; ZSCHOCKE, 2006).

- Metodología del CIAT (Centro Internacional de Agricultura Tropical), (CIAT. PROYECTO DE DESARROLLO $\mathrm{DE}$ AGROEMPRESAS RURALES, 2003).

- Metodología de CODESPA (FUNDACIÓN CODESPA, 2011).

\subsection{Metodología RURALTER}

Esta guía (Plataforma RURALTER, 2004) es el resultado de una construcción colectiva entre los colaboradores de la Plataforma RURALTER y a las experiencias de diversas instituciones de desarrollo en el análisis de cadenas productivas en el Perú, Ecuador y Bolivia. El trabajo empezó a mediados del año 2002, en el marco de la mesa de desarrollo económico de la Plataforma RURALTER. En esta mesa participaron profesionales de las instituciones Centro Internacional de Cooperación para el Desarrollo Agrícola (CICDA), Intercooperation (IC) y el Servicio Holandés de Cooperación al Desarrollo (SNV).

En esta guía se presenta el análisis de cadenas productivas en tres fases sucesivas (Figura 2):

1. Fase Preliminar: precisa el objetivo del análisis, la selección 
del producto de interés y los alcances del estudio. Es una fase de preparación y de delimitación.

2. Fase Central: abarca la recopilación, sistematización $\mathrm{y}$ ordenamiento de los datos. Se divide en seis bloques temáticos que permiten una clasificación ordenada de la información.

3. Fase Final: corresponde a los momentos de análisis de la información, identificación de los puntos críticos y ventajas competitivas de la cadena, con la finalidad de formular estrategias de acción.

\section{FIGURA 2: ANÁLISIS DE CADENAS PRODUCTIVAS EN TRES FASES SUCE- SIVAS}

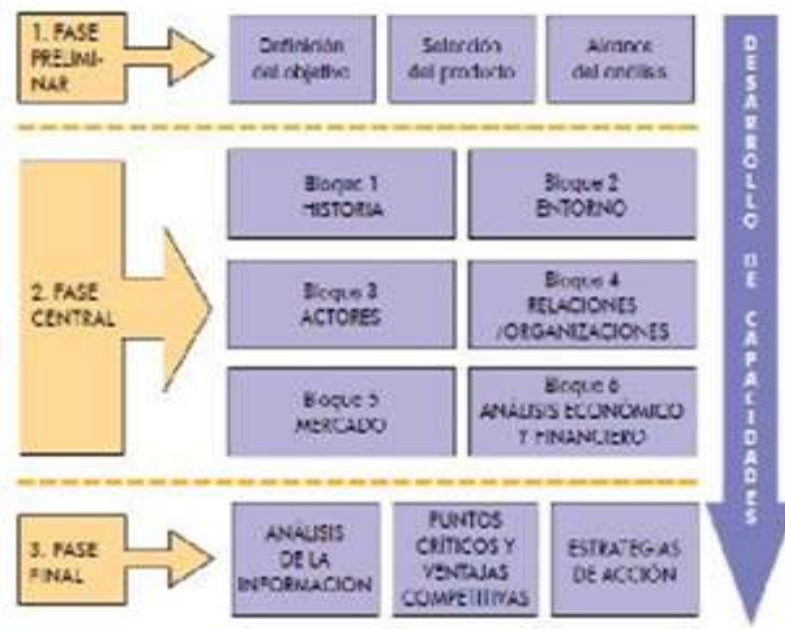

Fuente: Plataforma RURALTER (2004)

En las fases preliminar y central, se plantea responder 18 preguntas clave que permitirían orientar en el proceso de preparación, recolección y sistematización de la información.
La fase central está estructurada en seis bloques temáticos que ayudarían a recolectar, ordenar y sistematizar la información por tema: historia, entorno, actores, relaciones/organizaciones, mercado, análisis económico y financiero.

Se presentan 35 herramientas (de recolección de información, de análisis y de visualización de la Información) que permitirían a los usuarios recopilar y ordenar más fácilmente la información de los diferentes bloques.

La guía también propone varios ejemplos tomados de la realidad para ilustrar situaciones que surgen en los procesos de análisis de cadenas productivas.

Para la aplicación de la guía, se recomienda conformar un equipo de trabajo interinstitucional y multidisciplinario. La guía metodológica menciona varios métodos de recolección y análisis de la información a lo largo del documento, pero no detalla cómo se desarrollan estos métodos.

Dentro de los métodos propuestos para cada fase del análisis de cadenas productivas:

\section{Fase Preliminar:}

- Reuniones del equipo de trabajo y otras personas clave;

- Visitas al campo, observaciones ;

- Entrevistas con personas clave;

- Revisión de información secundaria.

\section{Fase Central:}

- Talleres participativos con productores; 


\section{ReLAlnEP}

- Talleres participativos con todos los actores locales (productores, comerciantes);

- Entrevistas con actores (productores, transformadores, comerciantes, servicios de apoyo) ;

- Revisión de información secundaria (mercado, políticas, censos);

- Paneles de expertos.

\section{Fase Final:}

- Reuniones del equipo de trabajo y personas clave para el análisis de la información;

- Exposiciones;

- Talleres participativos con todos los actores de la cadena para la definición de estrategias de acción.

\subsection{Metodología CIP (Centro Interna- cional de la Papa)}

El CIP ha desarrollado el "Enfoque Participativo en Cadenas Productivas (EPCP)" para definir, analizar e implementar oportunidades de negocio en conjunto con los diferentes actores de la cadena.

El EPCP es una herramienta participativa que fomenta la interacción entre diferentes actores de la cadena productiva con el fin de estimular innovaciones entre todos los actores de una cadena productiva. Bien guiado y estructurado, el EPCP gradualmente genera (a) interés, (b) confianza, y (c) colaboración entre los actores que participen en este proceso participativo. Las innovaciones que engendra pueden ser nuevos productos o procesos, nuevas tecnologías o nuevas instituciones, que tengan en común el beneficiar a los diferentes actores de la cadena directa o indirectamente.

El EPCP es un método flexible a ser aplicado en diferentes contextos o cadenas productivas. Su uso no se restringe a la agricultura solamente. De todos modos, la institución que aplica el método deberá adaptar el EPCP al contexto dado tomando en cuenta los factores que mejoran y arriesgan la obtención del impacto deseado, como por ejemplo reducción de pobreza, empoderamiento del agricultor, efectos favorables en género y mejoramiento ambiental.

El único elemento fijo de este método participativo son sus tres fases, cuya duración es flexible, dependiendo del avance del proceso. Cada fase tiene un objetivo específico y un evento de cierre, donde los resultados y futuros pasos son presentados y discutidos con un grupo más grande de participantes.

Es crucial que la institución que lidera el EPCP entienda la "lógica de sostenibilidad" que está detrás de estas tres fases, dejando paulatinamente a los actores claves asumir la influencia en la toma de decisiones mientras que esta institución asume más un rol facilitador. 


\section{FIGURA 3: METODOLOGÍA CIP}

Objetivo por Fase

$\widehat{~ F a s e ~} 1$

Conocer a los diferentes actores de la cadena, con sus actividades, intereses, problemas, ideas, etc.

Evento 1

Fase 2

Analizar de manera participativa el potencial de oportunidades de negocio

Evento 2

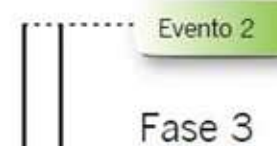

Estimular innovaciones compartidas

Evento fina
Participantes
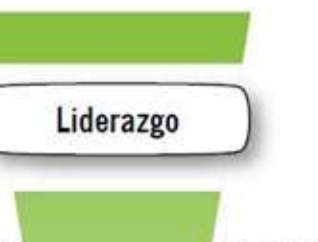

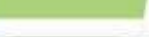

Facilitación

Confianza
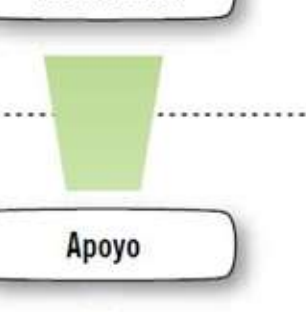

Fuente: Bernet, Thiele e Zschocke (2006)

Fase 1: del EPCP es un período de investigación diagnóstica. Generalmente toma de dos a tres meses e involucra entre 20 y 40 entrevistas cualitativas. En contraste con los estudios de diagnóstico convencionales, EPCP no enfatiza tanto en la recopilación y la evaluación de información técnica sino más bien en la identificación de los actores claves de la cadena productiva para conocer sus intereses, problemas y propuestas de solución. Los contactos establecidos en las entrevistas ayudan a interesar y motivar a los actores a participar en el primer evento del proyecto.

Para el evento de clausura de la Fase 1 se invita a las personas entrevistadas, otros actores claves de la cadena productiva y representantes de instituciones de investigación y del go- bierno. En una primera parte del evento, los hallazgos de las entrevistas son presentados y discutidos en pleno. Luego dos o tres grupos de trabajo son formados basados en áreas temáticas, ligadas en oportunidades de negocio, que han sido identificadas a lo largo de las entrevistas.

Estos grupos temáticos son los elementos claves de la Fase 2, ya que proveen un marco de trabajo de interacciones fructíferas donde se produce aprendizaje mutuo y se genera mayores niveles de confianza.

Fase 2: comienza con reuniones temáticas grupales que tratan desde un comienzo con el análisis de oportunidades potenciales de negocios. Para cada grupo temático la institución promotora provee un facilitador cuya tarea es 
la de preparar y guiar las diferentes reuniones. Es responsabilidad de esta persona estimular la buena participación y generar compromisos óptimos en las discusiones de grupo que requieren un fuerte enfoque hacia la demanda. El facilitador debe asegurar que las oportunidades potenciales de mercado sean correctamente identificadas y evaluadas, sin dar cabida a las interminables discusiones que vienen de un enfoque productivista.

Unas 6 a 10 reuniones por grupo serían suficientes para analizar cuidadosamente las diferentes oportunidades. En el proceso la institución líder puede contratar especialistas que apoyen a los grupos de trabajo con estudios de mercado.

En el evento final de esta fase, las oportunidades de mercado son identificadas, en las cuales participan los miembros de cada grupo, son presentadas por cada grupo de trabajo y discutidas en plenaria. Este evento provee un momento conveniente para invitar e integrar a nuevos actores para fortalecer a los grupos de trabajo con nuevos conocimientos y capacidades.

Fase 3: del EPCP se concentra en la implementación de las actividades que respondan a las oportunidades de mercado identificadas. El tipo de actividades y el tiempo necesario para su implementación puede variar según los diferentes grupos de trabajo y proyectos: situaciones complejas requieren más tiempo, mientras que reuniones frecuentes aceleran el proceso.

En cualquier caso, un periodo de tres a seis meses será necesario para implementar satisfactoria-mente las actividades planeadas. Lue- go en el evento de clausura de la Fase 3, cuando el EPCP termina, se presentan al público las innovaciones generadas, que pueden ser comerciales, organizacionales o de proceso, como información que ayuda a los diferentes actores a tomar mejores decisiones. A diferencia de los eventos previos, este último gran evento es orientado hacia la prensa, políticos y donantes. La idea es "capitalizar" todos los logros y echar luz a los actores claves que han participado en el proceso. Así se busca empoderar y dar continuidad a las innovaciones generadas.

El EPCP explícitamente termina con el gran evento de clausura de la Fase 3. Es importante entregar la responsabilidad completa a los actores de la cadena quienes son los dueños de las innovaciones generadas. Esto no quiere decir que la institución promotora no debe seguir apoyando a ciertas actividades luego para consolidar los productos innovados, procesos, e instituciones.

Sin embargo, la institución líder debe tener mucho cuidado en no crear dependencias que arriesgan un manejo exitoso de las innovaciones por los propios actores. El grado de apoyo y el conjunto de actividades requeridos variarán según el caso, dependiendo de la naturaleza de las innovaciones y las capacidades de los actores. El apoyo adicional será aún más necesario cuando se trata de innovaciones institucionales.

\subsection{Metodología CIAT (Centro Interna- cional de Agricultura Tropical)}




\section{ReLAInEPY}

El CIAT propone una metodología que se organiza en tres etapas para diseñar una estrategia con el objetivo de aumentar la competitividad de una cadena que involucra la participación de productores de pequeña escala; aporta un completo manual de campo que ofrece una serie de "preguntas de facilitación" para el análisis de la cadena.

\section{FIGURA 4: METODOLOGIA CIAT}

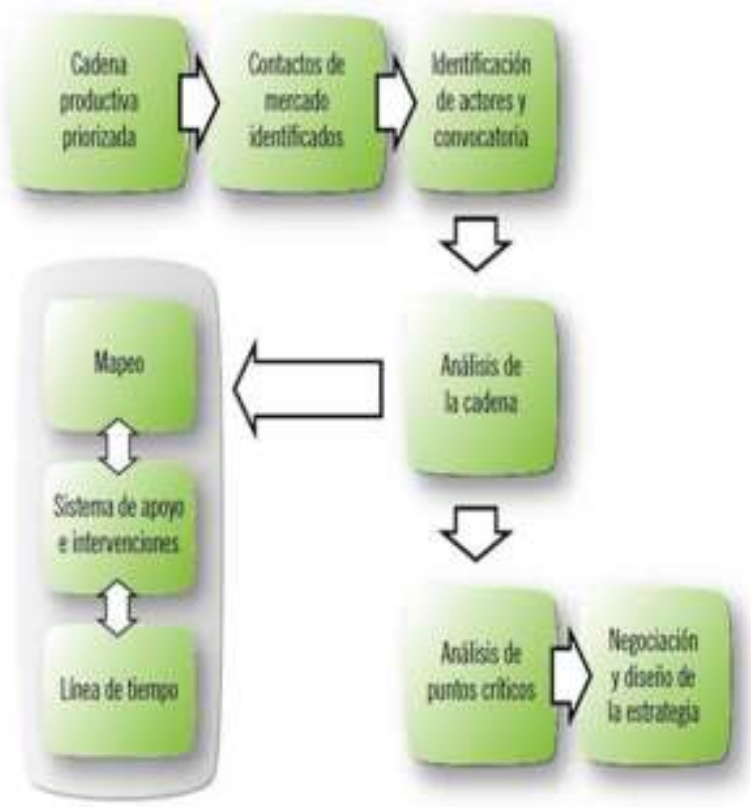

Fuente: CIAT. Proyecto de Desarrollo de Agroempresas rurales (2003)

Primera etapa: Se identifican y se aplican los criterios para seleccionar cadenas y con la cadena identificada se analizan los contactos comerciales existentes y potenciales y se revisan los actores para identificar y convocar los claves para el proceso.

Herramientas: árbol de decisiones; sondeo rápido de mercado; talleres.

Segunda etapa: Se analiza la cadena utilizando métodos como el mapeo, la revisión de la oferta y demanda de servicios de desarrollos empresariales disponibles, la generación de una línea de tiempo, la identificación y priorización de puntos críticos en cada eslabón y la generación de árboles de problemas y soluciones para la cadena como sistema. Además se compara la cadena con la competencia por medio de un proceso sencillo de benchmarking y se lleva a cabo un análisis prospectivo del mercado.

Herramientas: mapeo de la cadena; oferta de servicios de apoyo; línea de tiempo.

Tercera etapa: consiste en el análisis de puntos críticos donde la competitividad de la cadena enfrenta limitaciones, bien sea por factores internos o externos, cómo analizar las causas y efectos de estas limitaciones y da herramientas para pasar de las limitaciones a acciones positivas.

La entidad facilitadora sistematiza los resultados de los talleres de diagnóstico de la cadena y genera un documento técnico al respecto. Este documento sirve como insumo para el taller de negociación y diseño de la estrategia y se definen acciones para su ejecución a corto, mediano y largo plazo. Esta etapa finaliza con la elaboración de un camino lógico encaminado a fortalecer la competitividad de la cadena como sistema.

Herramientas: lluvia de ideas para identificar factores limitantes; matriz de doble entrada; árboles de problema por limitación prioritaria; árbol de problema general para la cadena; árbol de soluciones; taller de negociación.

Finalizadas las etapas se debe diseñar un sistema de monitoreo y evaluación de la implementación de estrategias de competitividad. 


\section{ReLAInEP}

Ventajas de la metodología: Sencillo, de aplicación rápida y gran carácter participativo.

\subsection{Metodología CODESPA}

Toma como base las metodologías de análisis de servicios en cadenas productivas y la metodología de análisis de cadenas productivas desarrollada por el CIAT (CIAT, 2003) y le aporta un enfoque de cadenas de valor (Figura $5)$.
La metodología de análisis con enfoque de cadena de valor se compone de seis fases:

1. Análisis del mercado potencial para el/los producto/s;

2. Mapeo de la cadena y análisis de los puntos críticos;

3. Análisis de los servicios de desarrollo empresarial (SDE) existentes

4. Prospectiva de la cadena;

5. Comparación entre la oferta y demanda de SDE en el territorio;

6. Diseño del plan de acción.

\section{FIGURA 5: METODOLOGÍA CODESPA}

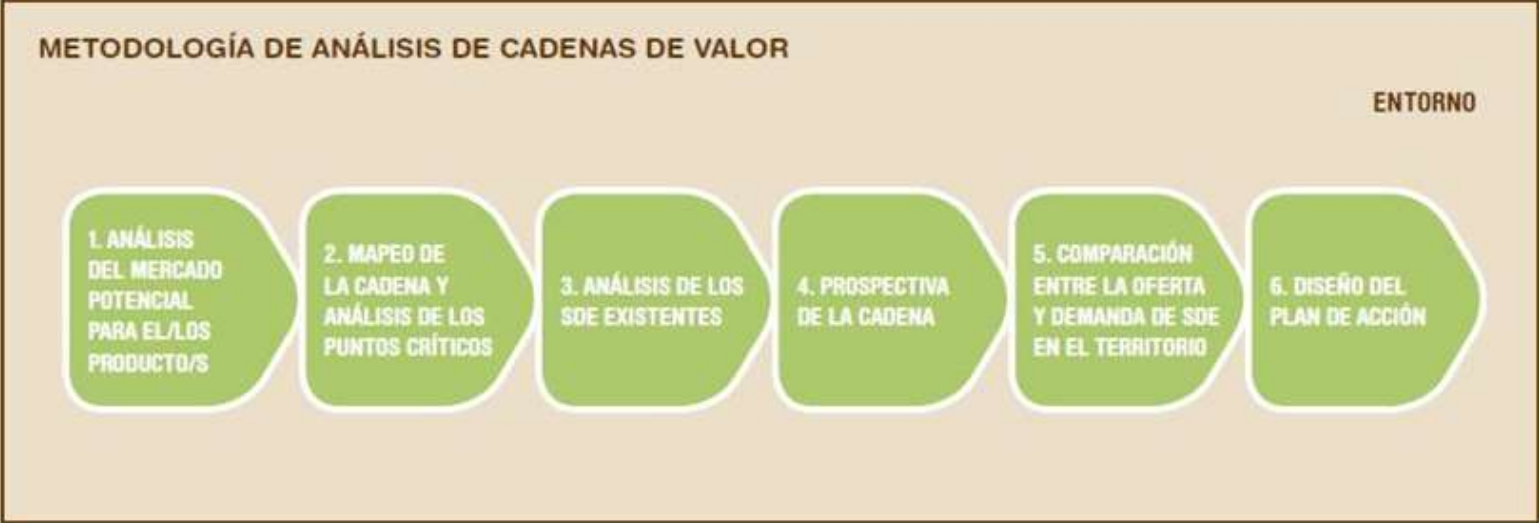

Fonte: Fundación CODESPA (2011)

El análisis de la metodología parte de revisar y analizar las opciones de mercado existentes para el producto de la cadena en cuestión, elemento central en la evolución al enfoque de cadenas de valor. Si no se dispone de estas opciones de mercado concretas, se debe resolver este punto de forma inmediata dado que sino la evolución hacia una cadena de valor va a llevar más tiempo o será prácticamente imposible.
Este análisis, a su vez, permite identificar y realizar un análisis de la cadena de suministro de todos los eslabones y actores de la cadena, lo cual corresponde al punto de mapeo y caracterización de la cadena, incluyendo un mapeo de los Servicios de Desarrollo Empresarial (SDE) existentes.

A continuación, el análisis se concentra en la visualización del futuro deseado por los actores de la cadena, mediante un ejercicio de prospección de mercados y productos, así como los 
SDE requeridos para alcanzar tales objetivos.

Estos objetivos y acciones constituyen la base del plan de acción de la cadena de valor.

Finalmente, se confronta la demanda de SDE requeridos con la oferta de las entidades de apoyo existentes en el territorio para determinar si son suficientes para atender tales demandas o si no existe una oferta de SDE disponible. En cualquiera de los dos casos, se definen estrategias para mejorar o desarrollar la oferta de SDE, los cuales son parte de la estrategia global para el desarrollo de la cadena de valor.
Toda esta información y resultados de los talleres se materializarán en un documento de análisis, el cual, junto con las recomendaciones y las conclusiones servirá para evolucionar hacia un trabajo bajo el enfoque de cadenas de valor. Al final, se desarrolla una última etapa destinada a esbozar un plan de acción, que contiene fundamentalmente un diseño preliminar de las estrategias para mejorar las cadenas productivas integrando el enfoque de cadenas de valor. Herramientas utilizadas en cada fase (Cuadro 2).

\section{CUADRO 2: DISTINCIÓN ENTRE CADENAS PRODUCTIVAS Y EL ENFOQUE DE CA-DENAS DE} VALOR

\begin{tabular}{|c|c|c|}
\hline FASE & HERRAMIENTA & MOMENTO \\
\hline $\begin{array}{l}\text { 1. Análisis del mercado potencial } \\
\text { para el/los producto/s }\end{array}$ & $\begin{array}{l}\text { Sondeo rapido de mercados (SRM) } \\
\text { Elaboracion de un portafolio de clientes }\end{array}$ & $\begin{array}{l}\text { Antes de los talleres de analisis de } \\
\text { cadena }\end{array}$ \\
\hline $\begin{array}{l}\text { 2. Mapeo de la cadena y análisis de } \\
\text { los puntos críticos }\end{array}$ & Mapas parlantes o diagramas & Durante el taller de analisis de cadena \\
\hline 3. Análisis de los SDE existentes & Matriz de servicios ofrecidos y recibidos & $\begin{array}{l}\text { Una parte durante el taller de analisis de } \\
\text { cadena y otra parte antes de éste }\end{array}$ \\
\hline 4. Prospectiva de la cadena & $\begin{array}{l}\text { Árbol de problemas } \\
\text { Vision de futuro } \\
\text { FODA o DOFA }\end{array}$ & Durante el taller de analisis de cadena \\
\hline $\begin{array}{l}\text { 5. Comparación entre la oferta y } \\
\text { demanda de SDE en el territorio }\end{array}$ & $\begin{array}{l}\text { Matriz de servicios ofrecidos versus } \\
\text { demanda de SDE de la cadena } \\
\text { proyectada, lluvia de ideas }\end{array}$ & $\begin{array}{l}\text { Una parte durante el taller de analisis de } \\
\text { cadena y otra posterior }\end{array}$ \\
\hline $\begin{array}{l}\text { 6. Diseño del plan de acción de la } \\
\text { cadena de valor }\end{array}$ & Plan de accion & $\begin{array}{l}\text { Una parte durante el taller de análisis de } \\
\text { cadena y otra posterior }\end{array}$ \\
\hline
\end{tabular}

Fuente: Fundación CODESPA (2011)

\section{Resultados: Cuadro Comparativo de}

\section{Metodologías}

$\mathrm{Si}$ bien las metodologías descriptas fueron creadas para su aplicación en el área agrícolarural, parecerían ser métodos flexibles que podrían ser aplicados en diferentes contextos o cadenas productivas. En particular, (BERNET; THIELE; ZSCHOCKE, 2006) aclara que la aplicación de su metodología no se restringe a la agricultura solamente.

Cada metodología, presenta procesos similares, son todas metodologías con carácter participativo, pero difieren en el tipo de herramientas utilizadas, los recursos necesarios para su aplicación y la presentación de las conclusiones obtenidas del análisis, entre otros. 
A los efectos de realizar un análisis comparativo se tuvieron en cuenta las siguientes variables:

- Cantidad de Pasos para su aplicación

- Nivel de Profundidad de la información obtenida.

- Cantidad de Herramientas sugeridas;
- $\quad$ Tiempo Total Requerido para su aplicación;

- $\quad$ Aplicaciones prácticas relevadas.

- A continuación se presenta un Cuadro 3 comparativo de las metodologías relevadas.

\section{CUADRO 3: COMPARATIVO DE LAS METODOLOGÍAS RELEVADAS}

\begin{tabular}{|c|c|c|c|c|}
\hline \multirow{8}{*}{$\begin{array}{l}\text { Cantidad de } \\
\text { Pasos }\end{array}$} & RURALTER & CIP & CIAT & CODESPA \\
\hline & 3 Fases & 3 Fases & 3 Etapas & 6 Fases \\
\hline & $\begin{array}{l}\text { Fase preliminar: } \\
\text { Definición objetivos, } \\
\text { producto y alcance } \\
\text { del }\end{array}$ & $\begin{array}{l}\text { Fase 1: Investigación } \\
\text { diagnóstica ( } 2 \text { a } 4 \\
\text { meses) }\end{array}$ & $\begin{array}{l}\text { Etapa 1: Selec- } \\
\text { ción cadena prio- } \\
\text { rizada, identifica- } \\
\text { ción y }\end{array}$ & $\begin{array}{l}\text { Fase 1: Análisis del } \\
\text { mercado. }\end{array}$ \\
\hline & $\begin{array}{l}\text { Fase central: recopi- } \\
\text { lación, sistematiza- } \\
\text { ción y }\end{array}$ & $\begin{array}{l}\text { Fase 2: Análisis de } \\
\text { oportunidades poten- } \\
\text { ciales de }\end{array}$ & $\begin{array}{l}\text { Etapa 2: Análisis } \\
\text { de la cadena ( } 3 \text { a } \\
4 \text { meses) }\end{array}$ & $\begin{array}{l}\text { Fase 2: Mapeo y } \\
\text { análisis de puntos } \\
\text { críticos. }\end{array}$ \\
\hline & \multirow{4}{*}{$\begin{array}{l}\text { Fase Final: Análisis } \\
\text { de información, iden- } \\
\text { tificación de }\end{array}$} & \multirow{4}{*}{$\begin{array}{l}\text { Fase 3: Implementa- } \\
\text { ción de las activida- } \\
\text { des (4 a } 6 \text { meses) }\end{array}$} & \multirow{4}{*}{$\begin{array}{l}\text { Etapa 3: Análisis } \\
\text { de puntos críticos, } \\
\text { negociación y } \\
\text { diseño }\end{array}$} & $\begin{array}{l}\text { Fase 3: Análisis de } \\
\text { SDE }\end{array}$ \\
\hline & & & & $\begin{array}{l}\text { Fase 4: Prospectiva } \\
\text { de la cadena. }\end{array}$ \\
\hline & & & & $\begin{array}{l}\text { Fase 5: comparati- } \\
\text { va entre oferta y } \\
\text { demanda de SDE. }\end{array}$ \\
\hline & & & & $\begin{array}{l}\text { Fase 6: Diseño plan } \\
\text { de acción }\end{array}$ \\
\hline $\begin{array}{l}\text { Nivel de pro- } \\
\text { fundidad }\end{array}$ & Alto & Medio & Medio & Medio \\
\hline $\begin{array}{l}\text { Número de } \\
\text { herramientas }\end{array}$ & $\begin{array}{l}\text { Alta (35 herramien- } \\
\text { tas) }\end{array}$ & Baja (7 herramientas) & Baja & $\begin{array}{l}\text { Baja (8 herramien- } \\
\text { tas) }\end{array}$ \\
\hline $\begin{array}{l}\text { Tiempo reque- } \\
\text { rido }\end{array}$ & Alto & Bajo (9 a 15 meses) & $\begin{array}{l}\text { Bajo (8 a } 12 \text { me- } \\
\text { ses) }\end{array}$ & Bajo \\
\hline $\begin{array}{l}\text { Aplicaciones } \\
\text { prácticas rele- } \\
\text { vadas }\end{array}$ & $\begin{array}{l}\text { Cadenas agroalimen- } \\
\text { tarias, lácteos, arroz, } \\
\text { brócoli. }\end{array}$ & Papa, vegetales & $\begin{array}{l}\text { Pollos, lácteos, } \\
\text { café }\end{array}$ & $\begin{array}{l}\text { Madera, hortalizas, } \\
\text { miel }\end{array}$ \\
\hline $\begin{array}{l}\text { Observaciones } \\
\text { Particulares }\end{array}$ & $\begin{array}{l}\text { Alto nivel de detalle } \\
\text { en la información } \\
\text { obtenida. La fase } \\
\text { central suele requerir } \\
\text { mucho tiempo }\end{array}$ & $\begin{array}{l}\text { Flexible (herramien- } \\
\text { tas } \\
\text { y tiempos), puede ser } \\
\text { aplicado a otros secto- } \\
\text { res. No enfatiza en la } \\
\text { recopilación y la } \\
\text { evaluación de la in- } \\
\text { formación técnica. }\end{array}$ & $\begin{array}{l}\text { Sencillo, rapidez } \\
\text { en su aplicación y } \\
\text { gran carácter } \\
\text { participativo }\end{array}$ & $\begin{array}{l}\text { Sencillo, rapidez en } \\
\text { su aplicación y } \\
\text { gran carácter parti- } \\
\text { cipativo. Permite } \\
\text { análisis de la cade- } \\
\text { na de suministro }\end{array}$ \\
\hline
\end{tabular}




\section{Conclusiones}

A través del relevamiento de publicaciones, papers, informes y casos se pudo tomar conocimiento de diversas metodologías de carácter participativo para el análisis de cadena productivas.

En varias oportunidades puede observarse que se utilizan los términos de cadena productiva, cadena de suministro y cadena de valor como sinónimos sin embargo parecería que existen diferencias sustanciales entre ellos. En este sentido podría decirse que la cadena productiva tiene un enfoque netamente descriptivo de la sucesión de operaciones necesarias para que un insumo se transforme y llegue en forma de producto a una consumidor final. La cadena de suministro y la administración de cadena de suministro parecieran hacer foco en términos de eficiencia, y variables como tiempo y costos óptimas. Por el contrario el concepto de cadena de valor pareciera hacer foco en el valor agregado para el cliente, beneficiando a todos los actores que la componen aun cuando no se cumplan determinados óptimos técnicos, por tratarse de una herramienta con visión estratégica.

- Respecto de la comparación de las metodologías analizadas puede concluirse lo siguiente:
- Cantidad de Pasos: mientras las tres primeras metodologías contemplan la realización de 3 fases o etapas, la metodología de CODESPA presenta 6 fases. Sin embargo, si se analiza en detalle el contenido de las etapas propuestas puede observarse que en realidad en esta última metodología se desdoblan actividades que en las otras metodologías están consolidadas.

- Nivel de Profundidad: Según la información que cada metodología permitiría relevar, es la propuesta por RURALTER aquella que tendría mayor profundidad de información relevada. Las metodologías de CIP, CIAT y CODESPA podrían clasificarse como de profundidad media.

- Cantidad de Herramientas: Como contrapartida al nivel de profundidad en la información, es la metodología de RURALTER aquella que también tiene la mayor cantidad de herramientas a aplicar, con más de 35 herramientas sugeridas, seguida por las otras tres con niveles similares de entre 7 y 8 herramientas sugeridas.

- Tiempo Requerido: El tiempo requerido para su aplicación es Alto en la metodología de RURALTER, ya que se estima que tomaría más de un año y medio la aplicación de la metodología completa. El resto de las 
herramientas, tomarían entre 8 y 15 meses para su aplicación.

- Aplicaciones Prácticas relevadas: Todas las metodologías relevadas muestran aplicaciones en el área agrícola-rural, con pequeños productores, dado su carácter participativo sin embargo no se descarta que puedan ser aplicadas a otras áreas y tipos de proyectos.

Por último, vale decir que deberían tenerse en cuenta diversos factores al momento de elegir una metodología de análisis participativo de cadenas productivas, tales como el sector de la economía sobre la cual se quiere intervenir, los recursos y tiempos disponibles y el acceso a los actores principales de la cadena, entre otros.

\section{Referências}

AYERS, J. B. Handbook of Supply Chain Management. St. Lucie Press, 2000.

BERNET, T.; THIELE, G.; ZSCHOCKE, T. Participatory Market Chain Aproach (PMCA). User Guide. Lima: International Potato Center (CIP) - Papa Andina, 2006.

BLANCHARD, D. Supply Chain Management Best Practices. $2^{\circ}$ Edition. John Wiley \& Sons, 2010.

CEDILlO, M. G.; SANCHEZ, J.; SANCHEZ, C. (2006). The new relational schemas of interfirms cooperation: the case of the Coahuila automobile cluster in Mexico. International Journal of Automotive Technology and Management, v 6, n. 4. , 406-418, 2006.

CHOPRA, S.; MEINDL, P. Supply Chain Management. $3^{\circ}$ Edition. Pearson/Prentice Hall, 2006.

CIAT. Proyecto de Desarrollo de Agroempresas rurales. Diseño de Estrategias para Au- mentar la Competitividad de Cadenas Productivas con Productores de Pequeña Escala. Manual de Campo. Cali, 2003.

FUNDACIÓN CODESPA. Cadenas de Valor: creando vínculos comerciales para la erradicación de la pobreza. Madrid: Codespa, 2010.

FUNDACIÓN CODESPA. Metodología de análisis de cadenas productivas bajo el enfoque de cadenas de valor. Madrid: Codespa, 2011.

KAPLINSKY, R.; MORRIS, M. A handbook for value chain research. IDRC, 2000.

MITCHEL, J.; COLES, C.; KEANE, J. Upgrading along vale chains: Strategies for poverty reduction in Latin America. Comercio y Pobreza en Latinoamerica (COPLA), 2009.

PLATAFORMA RURALTER. Guia metodológica para el análisis de cadenas productivas. Lima: Línea Andina S.A.C, 2004.

PORTER, M. Competitive advantage: creating and sustaining superior performance. New York: The Free Press, 1985.

ROSS, D. Managing in a New Era: the performance advantage. APICS: American Production and Inventory Control Society, 1996.

SAUCEDO LOPEZ, R. Cadena de Suministro. Universidad Autonoma de Nuevo Leon. Secretaría Técnica ASOCAM. Intercooperation. ¿Cómo hacer análisis de cadenas? Metodologías y casos, 2001 . 